\title{
Massive stars beyond the Local Group
}

\author{
C. J. Evans ${ }^{1}$, F. Bresolin ${ }^{2}$, R.-P. Kudritzki² ${ }^{2}$ W. Gieren ${ }^{3}$ \\ and G. Pietrzyński ${ }^{3}$ \\ ${ }^{1}$ UK ATC, Royal Observatory, Blackford Hill, Edinburgh, EH9 3HJ, UK \\ email: cje@roe.ac.uk \\ ${ }^{2}$ Institute for Astronomy, 2680 Woodlawn Drive, Honolulu, HI 96822, USA \\ ${ }^{3}$ Universidad de Concepción, Departamento de Física, Casilla 160-C, Concepción, Chile
}

\begin{abstract}
The next generation of ground-based telescopes will give access to resolved stellar populations in previously unexplored environments. To highlight the potential for stellar spectroscopy using an extremely large telescope (ELT), we present data from an ongoing project with the Very Large Telescope (VLT) to calibrate a novel method of distance determination. An ELT would extend the reach of this method and would allow quantitative studies of individual stars well beyond the Local Group, sampling a wide range of galaxy morphologies and metallicities.
\end{abstract}

Keywords. galaxies: stellar content - galaxies, individual: NGC 3109 - instrumentation: spectrographs - stars: early-type

\section{Calibrating the distance scale with the VLT}

NGC 3109 is a dwarf irregular at the edge of the Local Group at a distance of $\sim 1.3$ Mpc (e.g. Minniti et al. 1999). Aside from the big spiral galaxies, it is one of the more massive members of the Local Group yet, because of its distance, relatively little is known of its spectral content. As part of the larger ARAUCARIA project (P.I. Gieren) we have obtained VLT-FORS spectroscopy of the most visually-luminous stars in NGC 3109. Such stars are A-type supergiants, the direct descendants of massive OB-type stars.

Kudritzki et al. (2003) have advanced a new distance indicator, the 'flux-weighted gravity-luminosity relationship' (FGLR). From analysis of late-B and early-A supergiants, the bolometric magnitude and flux-weighted gravity are found to be strongly correlated. These calibrations are now being refined from analysis of the NGC 3109 spectra and in other systems such as NGC 55 .

\section{Stellar distances with an ELT}

The flux-weighted gravity can be found from analysis of medium-resolution $(\mathrm{R} \sim 5000)$ spectroscopy. When combined with photometric data, we can obtain the bolometric magnitudes, thence distances for individual stars. These stars are intrinsically very luminous. So for ELTs the prime limiting-factor for observations becomes spatial resolution rather than brightness, i.e. dependent on the level of the adaptive optics (AO) correction. If AO can provide spatial resolution toward the diffraction limit for a $30-50 \mathrm{~m}$ ELT, this technique offers the chance to obtain distances for the outer members of galaxies in the Virgo/Fornax clusters and beyond. This would provide an independent comparison to other methods of distance determination, e.g. from Cepheids. 


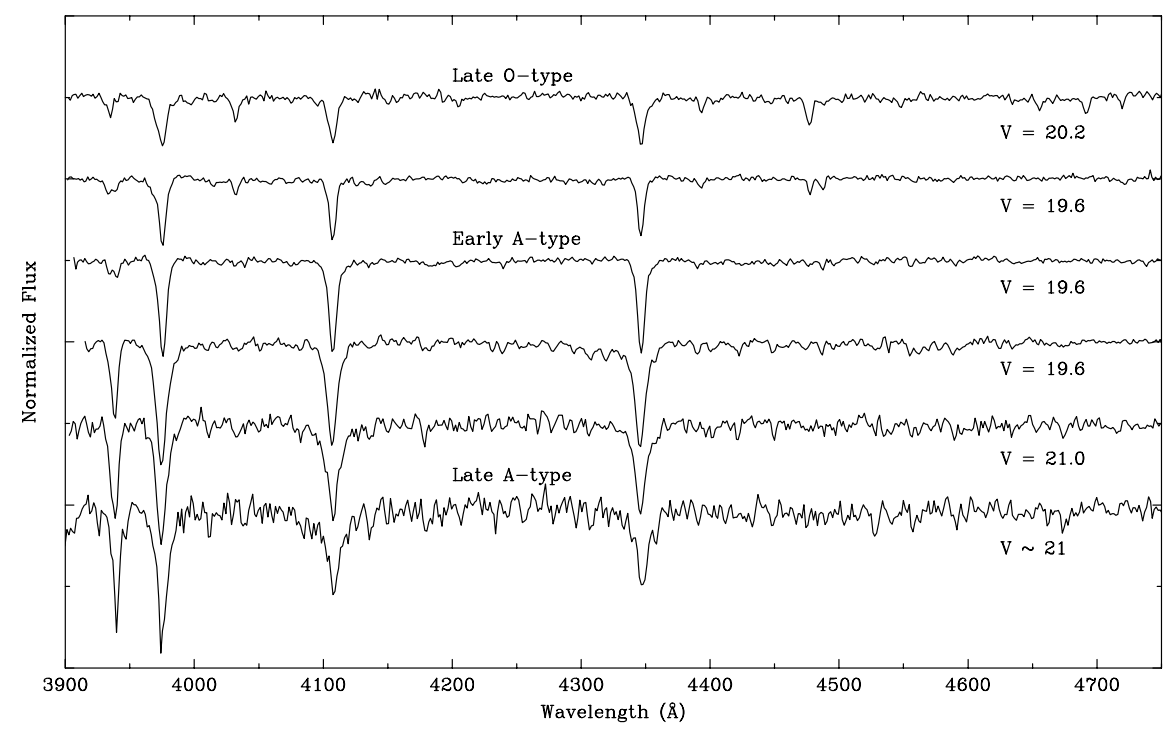

Figure 1. VLT-FORS spectroscopy of stars in NGC 3109.

\section{Instrumentation Requirements}

The FGLR method employs optical spectroscopy of the hydrogen Balmer lines, excluding $\mathrm{H} \alpha$, which can be strongly affected by stellar winds. Some of the requirements for instrumentation are:

- Spatial resolution/AO requirement: good encircled energy within 10-20mas

- Multiplex: $\sim 20$ objects (i.e. 2 or 3 pointings per galaxy)

- Spectral resolution: $\mathrm{R} \sim 5000$

- $\lambda$-coverage: $380-490 \mathrm{~nm}$

- Field-of-view: $\sim 2 \times 2$ arcmin

Obviously, with the best AO correction likely to be possible in the near-IR, theoretical modelling of redward diagnostics such as the Paschen lines in the $I$-band is clearly a strong priority for the stellar community.

\section{Other science drivers}

Exploiting the FGLR is just one project that illustrates the potential of ELT spectroscopy of resolved stellar populations. Other examples include:

- Exploring the high-mass population of 'local' galaxies (such as NGC 3109) at higherresolution $(\mathrm{R} \sim 20000)$ to determine chemical abundances etc.

- Studies of Wolf-Rayet stars out to $\sim 50 \mathrm{Mpc}$ and beyond, probing new environments and exploring the possible connection with gamma-ray bursts Vink \& de Koter 2005.

- IFU observations of resolved stellar clusters in, e.g. M82, to study the initial mass function (IMF) in starburst regions and the cluster mass function that modulates the IMF.

\section{References}

Kudritzki, R.-P., Bresolin, F., Przybilla, N. 2003, ApJ 582, L83

Minniti, D., Zijlstra, A.A., Alonso, M.V. 1999, AJ 117, 881

Vink, J.S. \& de Koter, A. 2005, A\& A 442, 587 